\title{
Time Programmed Feed of Semi-Batch Reactors with Non-Linear Radical Copolymerizations: An Experimental Study of the System Styrene + Divinylbenzene Using SEC/MALLS
}

\author{
Miguel A. D. Gonçalves, ${ }^{1}$ Rolando C. S. Dias, ${ }^{2}$ Mário Rui P. F. N. Costa*3
}

Summary: The radical crosslinking copolymerization of mono and divinyl monomers was experimentally studied with a $2.5 \mathrm{dm}^{3}$ semi-batch reactor using styrene + divinylbenzene as a model system. The analysis of products was carried out by SEC with a MALLS detector. The influence of the feed policy of divinylbenzene on the time evolution of the copolymer molecular weights and $z$-average mean square radius of gyration was assessed. A detailed kinetic model, in the absence of intramolecular reactions but taking into account the presence of the two isomers $\mathrm{m}$ - and $\mathrm{p}$ - in the commercial divinylbenzene and the different reactivities of the various radicals and double bonds was developed; most parameters have been collected from previous kinetic studies, and only two have been regressed using our measured molecular weights. These results can be used to improve the production of branched/crosslinked polymers with controlled molecular architecture.

Keywords: crosslinking; kinetic; modeling; semi-batch

\section{Introduction}

In recent years the synthesis and characterization of branched/crosslinked polymers has been a subject with increasing research activity. Indeed, there is much room for improving some properties of these materials as compared with their linear counterparts. Higher functional group densities and appropriated solubility and viscosity ranges can be exploited by producing hyperbranched polymers with different application fields (such as drug or gene delivery in biomedicine). In fact, for some

\footnotetext{
${ }^{1}$ LSRE-Instituto Politécnico de Bragança, Quinta de S. Apolónia, 5300 Bragança, Portugal Fax: (+351)273313051; E-mail: migueldiz@ipb.pt

2 LSRE-Instituto Politécnico de Bragança, Quinta de S. Apolónia, 5300 Bragança, Portugal Fax: (+351)273313051; E-mail: rdias@ipb.pt

3 LSRE-Faculdade de Engenharia da Universidade do Porto, Rua Roberto Frias s/n, Porto, 4200-465, Portugal

Fax: (+351)225081666; E-mail: mrcosta@fe.up.pt
}

application purposes, the highly ordered molecular architecture of dendrimers can be replaced by the less regular structure of hyperbranched polymers with economical gains due to the simplicity associated with the industrial production of the latter class of materials (usually a one-pot synthesis step). ${ }^{[1]}$ The synthesis of hyperbranched polymers is a well established subject for step-growth polymerization systems but less developed when vinyl monomers are involved. For this reason, in the latest few years, some works concerning the production of hyperbranched materials involving multi-functional vinyl monomers and using classical $^{[1-3]}$ or living ${ }^{[4]}$ radical polymerization have been published. A major problem of these polymerization systems is the occurrence of gelation for low monomer conversion even when a small amount of multi-functional vinyl monomer is used. In the aforementioned works ${ }^{[1-4]}$ some strategies have been put forward (such as the introduction of a chain transfer agent) to 
minimize this problem and make possible the production of soluble branched polymers at high monomer conversion. In another application field, copolymer networks are intentionally prepared by promoting the gelation of mono- and divinyl monomers, specially styrene + divinylbenzene. ${ }^{[5]}$ In this case, macroporous gel as insoluble swollen particles are formed, to be used as such for many separation processes in biotechnology and pharmaceutical industry (such as separation of albumin ${ }^{[6]}$ ), besides the well known application in polymer molecular size fractionation by SEC.

In the present work the copolymerization of styrene (S) with commercial divinylbenzene (DVB) in a semi-batch reactor has been investigated. An experimental confirmation of our previous theoretical work concerning the control of the crosslinked structure of the copolymer by adjusting the feed policy of the reagents ${ }^{[7]}$ has been achieved. The results here presented show it is possible to modify the production processes of branched/crosslinked polymers, namely with the purpose of obtaining soluble branched polymers at higher conversions than the conventional batch operation. A further benefit of this research was the possibility of having a better insight on the kinetics of these polymerization systems.

\section{Experimental Part}

Figure 1 depicts the experimental set-up used to carry out semi-batch crosslinking copolymerizations of styrene with divinylbenzene. The stainless steel reaction vessel (2.5 $\mathrm{dm}^{3}$ capacity) is equipped with a refrigeration jacket for keeping reaction temperature at the desired set-point. A thermocouple is used for on-line measurement of the temperature of the reaction medium. The control of the temperature is automatically executed by the refrigeration bath (heating/cooling) which is controlled by the computer. The time programmed feed of the monomers, initiator and chain transfer agent is possible thanks to the two metering pumps which are also computer controlled. A third pump operates at constant flow rate. The reactants to be fed into the reactor using the semi-batch mode are stored in three stainless steel reservoirs $\left(2 \times 1 \mathrm{dm}^{3}+0.2 \mathrm{dm}^{3}\right)$ kept at the same temperature as the reactor by using a heating bath. An argon stream is used to purge the contents of the reactor and is continuously bubbled through the polymerizing solution during the reaction. The reactor is equipped with a condenser in order to prevent monomer or solvent losses due to the bubbling process. The speed of the mechanical agitator is regulated using the computer.
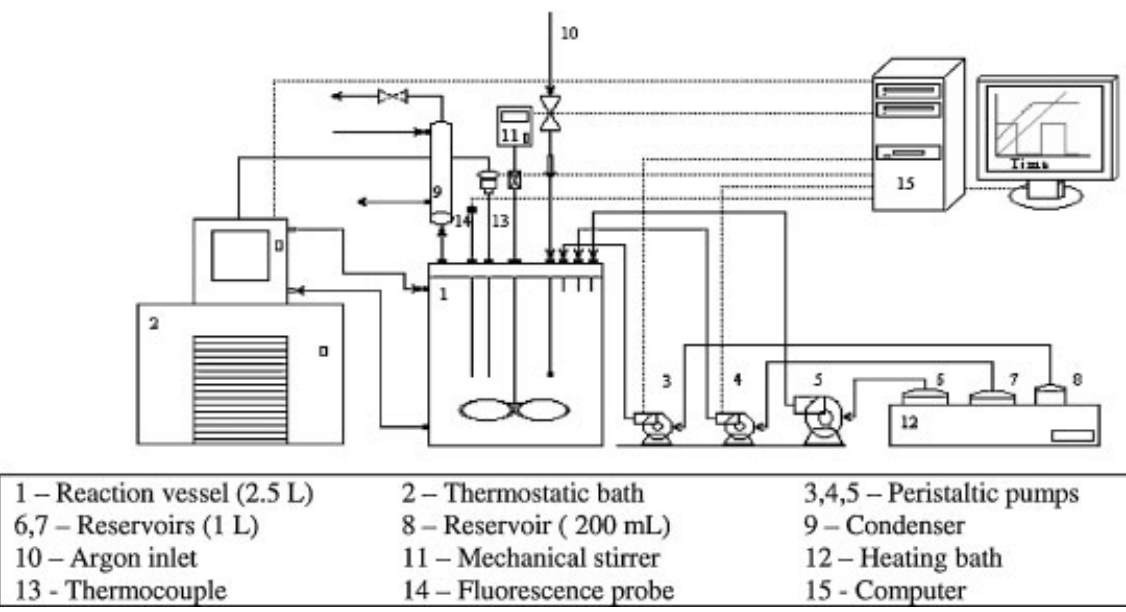

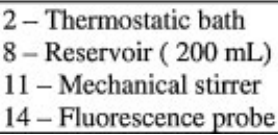

3,4,5 - Peristaltic pumps

9 - Condenser

12 - Heating bath

15 - Computer

Figure 1.

Experimental set-up used in the semi-batch crosslinking copolymerizations of S/DVB. 
Toluene at $99.7 \%$ purity, AIBN at $98 \%$ purity, styrene stabilized with $0.005 \% \mathrm{w} / \mathrm{w}$ 4-tert-butylcatechol at $99 \%$ purity and commercial grade divinylbenzene stabilized with $0.1 \%$ w/w 4-tert-butylcatechol at $80 \%$ purity have been purchased from Sigma Aldrich and used as received. That commercial DVB is a mixture of isomers, $56.2 \% \quad m$-divinylbenzene and $24.2 \%$ p-divinylbenzene, plus $19.6 \%$ of ethylvinylbenzene

The desired initial quantities of monomers and solvent were charged to the reaction vessel and brought up to the desired reaction temperature. The feeding reservoirs were charged at the same time with the needed amounts of reagents in order to implement the chosen feed policies. The feeding system was also brought up to the desired reaction temperature. Argon was bubbled in the reaction vessel at a flow rate of $40 \mathrm{~cm}^{3} /$ min during one hour, before the start of the polymerization in order to deoxygenate the reaction medium, and that same flow rate was kept during the whole polymerization process.

The polymerizations were started (defining the instant $t=0$ ) by adding the initiator (AIBN) to the reactor and the feeding system was immediately turned on. At prescribed polymerization times, samples of polymer were withdrawn from the reactor. For each sample, a portion was diluted in THF and analyzed in the SEC/ MALLS system and the remaining quantity was precipitated in methanol to be later also analyzed by SEC/MALLS.

Molecular weights and average molecular radius of gyration in THF were measured with a Polymer Laboratories PL-GPC-50 integrated SEC system with differential refractometer working at $950 \pm$ $30 \mathrm{~nm}$ attached to a Wyatt Technology DAWN8 $^{+}$HELEOS $658 \mathrm{~nm}$ Multi Angle Laser Light Scattering (MALLS) detector. The polymer samples were fractioned by molecular size using a train of 3 GPC columns PL gel $(300 \times 7.5 \mathrm{~mm})$ with nominal particle size $10 \mu \mathrm{m}$ and pore type MIXEDB-LS, maintained at constant temperature of $30{ }^{\circ} \mathrm{C}$ and using THF as the eluent at a flowrate of $1 \mathrm{ml} / \mathrm{min}$. A Wyatt Technology OPTILAB DSP $633 \mathrm{~nm}$ interferometric refractometer was used to measure the refractive index increment $(d n / d c)$ for the polymers, solvent and styrene in THF (respectively, 0.189, 0.110 and $0.170 \mathrm{~cm}^{3} / \mathrm{g}$ ), required for analyzing the MALLS results and for estimating the overall conversion from the values of the differential refractometer (RI) peak areas of monomer and polymer in the chromatographic traces of the SEC analysis. The accuracy of this latter method has been confirmed by gravimetry.

\section{Kinetic Modeling}

The modeling of the present case study was carried out using the general kinetic approach allowing the prediction of molecular weight and sequence distributions as well as the $z$-average molecular radius of gyration (for Gaussian chains) for nonlinear irreversible polymerization systems. ${ }^{[8-10]}$ Twenty nine different chemical species are considered as shown in Table 1. The copolymerization of styrene with commercial divinylbenzene (mixture of isomers), eventually in the presence of an inhibitor and/or a retarder (besides initiator, solvent and a chain transfer agent) is considered. Six different kinds of polymer radicals are distinguished since they present different structures and therefore different reactivities are also expected. ${ }^{[5,11-13]}$ Pendant double bonds arising from $m$ - and $p$-DVB, which are akin of two additional monomers in the present analysis, are also mutually distinguished as well as from the double bonds in the monomers as they are known to show different reactivities. ${ }^{[13]} \mathrm{A}$ discussion concerning the origins of the scatter in published values of the reactivities of these different species can be found in the same reference. ${ }^{[13]}$

In Table 2 the kinetic scheme considered for this polymerization system is presented. A total count of 114 chemical reactions is supposed to exist: initiator decomposition (1), initiation of monomers and PDB by primary radicals (15), propagation of monomers and PDB with the different kinds of polymer radicals (30), chain 
Table 1.

Chemical groups for the model of radical copolymerization of styrene (S) with divinylbenzene (DVB).

\begin{tabular}{|c|c|}
\hline Group Description & Alias \\
\hline \multicolumn{2}{|l|}{ Active groups in the polymer } \\
\hline Radical from styrene (RS) & $A_{1}$ \\
\hline Radical from m-divinylbenzene (RmDVB) & $\mathrm{A}_{2}$ \\
\hline Radical from p-divinylbenzene (RpDVB) & $\mathrm{A}_{3}$ \\
\hline Radical from m-pendant double bonds (RmPDB) & $\mathrm{A}_{4}$ \\
\hline Radical from p-pendant double bonds (RpPDB) & $\mathrm{A}_{5}$ \\
\hline Radical from Retarder (RR) & $A_{6}$ \\
\hline m-Pendant double bonds (m-PDB) & $\mathrm{M}_{4}$ \\
\hline p-Pendant double bonds ( $p$-PDB) & $\mathrm{M}_{5}$ \\
\hline \multicolumn{2}{|l|}{ Active groups/reagents not belonging to the polymer } \\
\hline Styrene $(\mathrm{S})$ & $M_{1}$ \\
\hline m-divinylbenzene (m-DVB) & $\mathrm{M}_{2}$ \\
\hline$p$-divinylbenzene ( $p$-DVB) & $\mathrm{M}_{3}$ \\
\hline Initiator (AIBN) (I) & $\mathrm{C}_{1}$ \\
\hline Solvent (Toluene) (T) & $\mathrm{C}_{2}$ \\
\hline Chain transfer agent (Carbon-tetrabromide) (CTA) & $\mathrm{C}_{3}$ \\
\hline Inhibitor (Z) & $\mathrm{C}_{4}$ \\
\hline Retarder (R) & $\mathrm{C}_{5}$ \\
\hline Primary radical from initiator (PRI) & $\mathrm{R}_{1}$ \\
\hline Primary radical from solvent (PRT) & $\mathrm{R}_{2}$ \\
\hline Primary radical from chain transfer agent (PRCTA) & $\mathrm{R}_{3}$ \\
\hline \multicolumn{2}{|l|}{ Inactive groups in the polymer } \\
\hline Polymerized styrene & $\mathrm{U}_{1}$ \\
\hline Polymerized m-divinylbenzene & $\mathrm{U}_{2}$ \\
\hline Polymerized p-divinylbenzene & $\mathrm{U}_{3}$ \\
\hline Crosslinking site from m-divinylbenzene & $\mathrm{U}_{4}$ \\
\hline Crosslinking site from p-divinylbenzene & $\mathrm{U}_{5}$ \\
\hline Fragments from initiator, solvent, chain transfer agent, inhibitor and retarder & $\mathrm{F}_{1}$ to $\mathrm{F}_{5}$ \\
\hline
\end{tabular}

transfers to solvent (6), chain transfers to CTA (6), inhibition of polymer and primary radicals (9), retardation of carbon centered polymer radicals (5), termination by combination of polymer radicals $(21)$, termina- tion by disproportionation of polymer radicals (21). Despite the difficult distinction between inhibitors (such as 4-tertbutylcatechol) and retarders (deactivation of primary radicals - deactivation/slowing

\section{Table 2.}

Kinetic scheme of radical copolymerization of styrene (S) + divinylbenzene (DVB).

\begin{tabular}{|c|c|}
\hline Kinetic steps & Chemical equation \\
\hline Initiator decomposition & $C_{1} \stackrel{k_{d}}{\longrightarrow} 2 f R_{1}$ \\
\hline Initiation of monomers and PDBs & $R_{j}+M_{k} \stackrel{k_{l j k}}{\longrightarrow} A_{k}+U_{k}+F_{j}$ \\
\hline Styrene propagation & $A_{i}+M_{1} \stackrel{k_{\text {pi1 }}}{\longrightarrow} A_{1}+U_{1}$ \\
\hline$m$-DVB and $p$-DVB propagations & $A_{i}+M_{j} \stackrel{k_{p i j}}{\longrightarrow} A_{j}+M_{2+j}+U_{j}$ \\
\hline$m-P D B$ and $p$-PDB propagations & $A_{i}+M_{j} \stackrel{k_{p i j}}{\longrightarrow} A_{j}+U_{j}$ \\
\hline Chain transfers to solvent & $A_{i}+C_{2} \stackrel{k_{S i}}{\longrightarrow}$ Dead end $+R_{2}$ \\
\hline Chain transfers to agent & $A_{i}+C_{3} \stackrel{k_{C T A i}}{\longrightarrow}$ Dead end $+R_{3}$ \\
\hline Inhibition of polymer radicals & $A_{i}+C_{4} \stackrel{k_{z i}}{\longrightarrow}$ Dead end $+F_{4}$ \\
\hline Inhibition of primary radicals & $R_{i}+C_{4} \stackrel{k_{z P i}}{\longrightarrow}$ Inactive Products \\
\hline Retardation of polymer radicals & $A_{i}+C_{5} \stackrel{k_{R i}}{\longrightarrow} A_{5}+F_{5}$ \\
\hline Termination by combination & $A_{i}+A_{j} \stackrel{k_{\text {tcij }}}{\longrightarrow}$ Head - Head Unit \\
\hline Termination by disproportionation & $A_{i}+A_{j} \stackrel{k_{t d i j}}{\longrightarrow}$ Sat. + Unsat. Units \\
\hline
\end{tabular}


of polymer radicals), the kinetic steps here considered involving these two species take into account the deactivation of all kinds of radicals and the existence of a polymer radical with a lower reactivity $\left(\mathrm{A}_{6}\right)$ coming from propagation with a retarder (such as oxygen). This radical is supposed to polymerize with monomers, as commonly accepted for oxygen centered radicals in styrene polymerization. ${ }^{[1]}$ Note that our experimental set-up has avoided this possible issue; simulations have shown that the effect of inhibitor on average molecular weights should only be visible above 500 ppm.

Termination in styrene polymerization is known to occur nearly always through radical combination, but disproportionation was included for sensitivity analysis purposes.
Table 3 and 4 present the numerical values of the 115 kinetic parameters used in the simulations. Past experimental works concerning the homopolymerization of styrene, ${ }^{[11]}$ pure $m$-DVB or pure $p$-DVB ${ }^{[12]}$ and the crosslinking of styrene with both pure DVB isomers and their mixture ${ }^{[13]}$ were used for estimating most of the needed kinetic parameters. Other assumptions are described in the footnotes of the aforementioned tables.

\section{Results and Discussion}

Table 5 describes the set of our experiments on the radical copolymerization of $\mathrm{S}$ with DVB in toluene solution at $60^{\circ} \mathrm{C}$. Linear polystyrene (A) and S/DVB without (B) and with CTA $(\mathbf{C})$ have been produced in a

Table 3.

Basic set of kinetic parameters considered in the modeling of the radical copolymerization of styrene (S) with divinylbenzene (DVB) at $60^{\circ} \mathrm{C}$.

\begin{tabular}{|c|c|c|}
\hline Kinetic step & Kinetic parameters involved ${ }^{\mathrm{a}}$ & Remarks \\
\hline AIBN decomposition & $k_{d}=9.6 \times 10^{-6}\left(\mathrm{~s}^{-1}\right), f_{0}=0.6(-)$ & b) \\
\hline Styrene homopropagation & $k_{p 11}=340$ & c) \\
\hline Styrene/m-, p-DVB propagations & $\begin{array}{l}r_{12}=k_{p 11} / k_{p 12}=0.43, k_{p 12}=790.70 \\
r_{13}=k_{p 11} / k_{p 13}=0.24, k_{p 13}=1416.67\end{array}$ & d) \\
\hline Styrene/m-, p-PDB propagations & $\begin{array}{l}r_{14}=k_{p 11} / k_{p 14}=0.92, k_{p 14}=369.57 \\
r_{15}=k_{p 11} / k_{p 15}=0.50, k_{p 15}=680\end{array}$ & d) \\
\hline m-, p-DVB homopropagation & $k_{p 22}=607.55, k_{p 33}=403.90$ & e) \\
\hline Polymer radicals termination & $\begin{array}{l}k_{p 11} / \sqrt{k_{t}}=0.035, k_{t}=9.44 \times 10^{7} \\
k_{t c}=\alpha_{t c} k_{t}, k_{t d}=\alpha_{t c} k_{t}\end{array}$ & f) \\
\hline Chain transfer to solvent (toluene) & $C_{S}=k_{S 1} / k_{p 11}=0.12 \times 10^{-4}$ & g) \\
\hline Chain transfer to agent $\left(\mathrm{CBr}_{4}\right)$ & $C_{C T A}=k_{C T A 1} / k_{p 11}=1$ & h) \\
\hline Inhibition & $C_{Z}=k_{Z 1} / k_{p 11}=520$ & i) \\
\hline Reaction radical/retarder & $k_{R 1}=10^{9}$ & j) \\
\hline
\end{tabular}

a) Kinetic parameters expressed in $\mathrm{dm}^{3} \mathrm{~mol}^{-1} \mathrm{~s}^{-1}$, unless otherwise stated.

b) $k_{d}$ collected from Ref. [11] (p. 71) and $f_{0}$ from Ref. [15].

c) IUPAC benchmark value[11] collected from Ref. [16]. ${ }^{\text {d) }}$ Reactivity ratios collected from Ref. [13].

e) Based on the experimental evaluation of the parameter $k_{p} / \sqrt{k_{t}}$ for the homopolymerizations of $m$-divinylbenzene and $p$-divinylbenzene[12] at $70^{\circ} \mathrm{C}$. Activation energies for propagation $\left(E_{p}=\right.$ $\left.32.5 \mathrm{kJmol}^{-1}\right)^{[11]}$ and for termination $\left(E_{t}=7.03 \mathrm{kJmol}^{-1}\right)^{[17]}$ were used for estimate this parameter at $60^{\circ} \mathrm{C}$. An overall average termination constant $\left(k_{t}\right)$ was considered in these calculations ${ }^{f}$.

${ }^{\mathrm{ff}} k_{t}$ is an average termination constant in the framework of the classical kinetics. Scattered values in the range 0.01 to 0.04 for the parameter $k_{p} / \sqrt{k_{t}}$ can be found in the literature.[5,17-20] The value $k_{p} / \sqrt{k_{t}}=0.035\left(\mathrm{dm}^{3} /\right.$ $(\mathrm{mol} \mathrm{s}))^{1 / 2}$ was estimated from time/conversion data (prior to noticeable Norrish-Trommsdorff effect) as measured in the present work. Termination of styrene is generally accepted to occur by combination $\left(\alpha_{t c}=1\right)$. Nevertheless values of $k_{t d} / k_{t c}$ up to 0.2 are also mentioned in the literature.[11] Experimental data of the present work are also consistent with $\alpha_{\text {tc }}$ close to 1 .

g) Parameter correspondent to the pair styrene/toluene. ${ }^{[1]}$

h) Scattered values for the pair styrene/ $\mathrm{CBr}_{4}$ are reported.[17]: the experimental results of the present work are consistent with a transfer constant close to ideality $\left(C_{C T A}=1\right)$ as also reported in the literature. ${ }^{[1]}$

i) Parameter correspondent to the pair styrene/p-benzoquinone. ${ }^{[1]}$

${ }^{j)}$ In the range of the rate constant for reaction of carbon-centered radicals with oxygen. ${ }^{[1]}$ 


\section{Table 4.}

Assumptions used in the present work for some kinetic parameters considered in the radical copolymerization of styrene (S) with divinylbenzene (DVB) at $60^{\circ} \mathrm{C}$.

\begin{tabular}{|c|c|c|}
\hline Kinetic step & Kinetic parameters involved & Remarks \\
\hline Propagation of S, DVB and PDB with radicals $A_{2}$ to $A_{5}$ & Geometric decay & a) \\
\hline Propagations with radicals RR & $k_{p 6 j} / k_{p 1 j}=0.01(j=1, \ldots, 5)$ & b) \\
\hline Initiations & $k_{l k j}=k_{p 1 j}(k=1, \ldots, 3, j=1, \ldots, 5)$ & c) \\
\hline Chain transfers to agent and to solvent & $k_{C T A i}=C_{C T A} k_{p i 1}, k_{S i}=C_{S} k_{p i 1}$ & d) \\
\hline Inhibition & $\begin{array}{l}k_{Z i}=C_{Z} k_{p i 1}(i=1, \ldots, 6) \\
k_{Z P i}=C_{Z} k_{p 11}(i=1, \ldots, 3)\end{array}$ & d) e) \\
\hline Reaction of radicals with retarder & $k_{R i} / k_{R 1}=k_{p i 1} / k_{p 11}(i=1, \ldots, 5)$ & d) \\
\hline Termination & $k_{\mathrm{tcij}}=k_{\mathrm{tc}}, k_{\mathrm{tdij}}=k_{\mathrm{td}}(i, j=1, \ldots, 6)$ & f) \\
\hline
\end{tabular}

a) It is considered that a decrease of reactivity of RmDVB relatively to RS occurs as inferred from experimental data leading to $k_{p 22} / k_{p 12}=0.77$. In the same conditions a lower reactivity ratio for RpDVB $\left(k_{p 33} / k_{p 13}=0.29\right)$ is estimated. It is postulated that RmPDB and RpPDB present a geometric reactivity drop relatively to RmDVB and $\operatorname{RpDVB}\left(0.77^{2}=0.59\right.$ and $0.29^{2}=0.08$, respectively). These reactivity decreases are plausible owing to steric factors. It has been previously shown[21] that only with a strong deviation from ideality a noticeable influence of these parameters in the gelation of the system is expected.

${ }^{b)}$ The propagation of the monomers with non-carbon centered radicals (e. g. oxygen centered) is considered to be much slower than with the correspondent normal propagation.[11]

${ }^{c}$ The rate constants for the initiation of the different monomers $(j=1, \ldots 5)$ with different kinds of primary radicals $(k=1, \ldots 3)$ are considered to take the same values as in the corresponding propagations with RS.

d) The different propagating radicals are considered to have similar reactivity decays for reactions with CTA, solvent, inhibitor and retarder.

e) The inhibition of the primary radicals is supposed to occur with RS at a same extent.

${ }^{\mathrm{f})}$ It is considered that the kinetic constants for all termination reactions take the same values as the average termination rate constant.

batch reactor. Semi-batch runs using different feed policies of DVB are described in experiments $\mathbf{D}, \mathbf{E}$ and $\mathbf{F}$ (a smaller concentration of AIBN was here used). A more or less constant global mole fraction of DVB was used in all batch and semi-batch experiments.

In Figure 2(a) are presented the experimentally measured and predicted values of global monomer conversion. Those data were used to estimate the parameter $k_{p} / \sqrt{k_{t}}=0.035 \mathrm{dm}^{3 / 2}(\mathrm{~mol} \mathrm{~s})^{-1 / 2}$ considering kinetic controlled polymerization ${ }^{[14]}$ for S/DVB copolymerization prior to gelation.
In Figure 2(b) are compared the experimental measurements of $\bar{M}_{w}$ for the nonlinear polymerization of S/DVB in batch reactor (run B) with the predictions obtained considering the set of kinetic parameters presented in Table 3 and 4. It can be observed that in these conditions $\left(r_{14}=0.92, r_{15}=0.50\right)$ a huge discrepancy between predictions and experimental values of $\bar{M}_{w}$ (and gelation time) occurs. Some of this discrepancy can be explained by the influence of the reaction medium, namely the effect of the solvent ${ }^{[12]}$ on the kinetics of these polymerization systems

\section{Table 5.}

Description of the set of experiments performed in the study of the radical copolymerization of S with DVB in toluene solution at $60^{\circ} \mathrm{C}$ in a semi-batch reactor.

\begin{tabular}{lcllll}
\hline Exp. & $\mathrm{S}_{\mathrm{O}}$ & DVB $_{\mathrm{O}}$ & AIBN $_{\mathrm{O}}$ & $\mathrm{T}_{\mathrm{O}}$ & \multicolumn{1}{c}{ Feed Conditions } \\
\hline A & 4.350 & 0.0 & 0.081 & 4.694 & Batch \\
B & 4.312 & 0.061 & 0.081 & 4.654 & Batch with $y_{D V B}=0.01395$ \\
C & 4.312 & 0.061 & 0.081 & 4.654 & Batch with $y_{D V B}=0.01395$ and mole ratio CTA/DVB $=0.08168$ \\
D & 4.350 & 0.0 & 0.081 & 4.694 & Constant feed rate $0.73 \mathrm{~mL} / \mathrm{min}$ during $2 \mathrm{~h}$. (final $y_{D V B}=0.01358$ ) \\
E & 4.350 & 0.0 & 0.081 & 4.694 & Constant feed rate $0.40 \mathrm{~mL} / \mathrm{min}$ during 6 h. (final $y_{D V B}=0.01371$ ) \\
F & 4.350 & 0.0 & 0.050 & 4.694 & Constant feed rate $0.65 \mathrm{~mL} / \mathrm{min}$ during $5.5 \mathrm{~h}$. (final $\left.y_{D V B}=0.01390\right)$ \\
\hline
\end{tabular}

${ }^{\text {a) }}$ Concentrations expressed in $\mathrm{mol} / \mathrm{dm}^{3}$. 


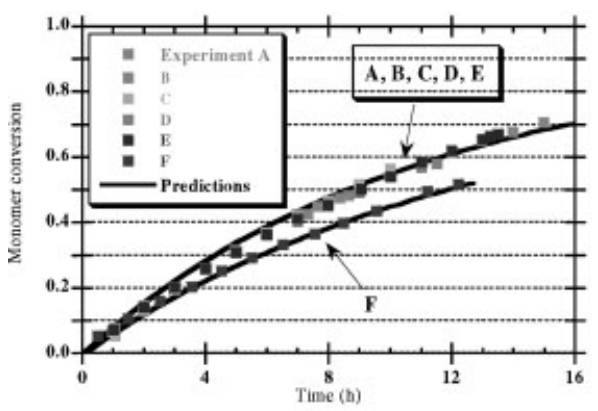

(a)

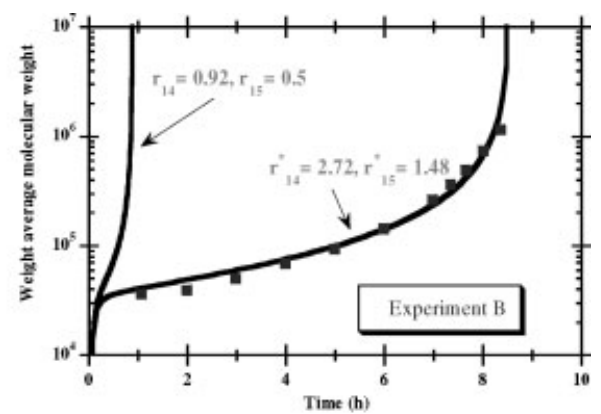

(b)

Figure 2.

Time evolution of the measured overall monomer conversion for linear and non-linear polymerization systems considering different operation conditions (a). The influence of the reactivity of the pendant double bonds on the predicted $\bar{M}_{w}$ (run B) and its comparison with the correspondent measured values (b).

(toluene in the present work vs. nearly bulk polymerization in Ref. $\left.{ }^{[13]}\right]$ ). However, the most important cause of the delay of gelation is likely to be the occurrence of cyclization (intramolecular) reactions..$^{[5,7]}$ For the sake of simplicity, in the present work, these combined effects are taken into in account by fitting apparent values of the reactivity of pendant double bonds, as they are the major parameters controlling the cross-linking process. It is also considered that the reactivity of $m$ - and $p$ - pendant double bonds is affected in the same proportion by these phenomena. Apparent values of reactivity ratios $r_{14}^{*}=2.72$ and $r_{15}^{*}=1.48$ were therefore estimated using the experimental information in run $\mathbf{B}$ (see

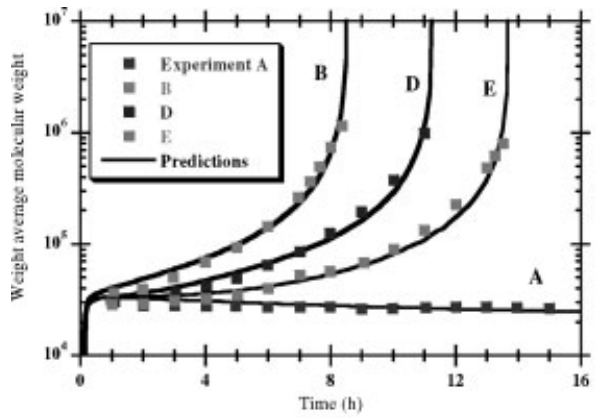

(a)
Figure 2(b)). Note that these apparent reactivity ratios are consistent with a decrease of reactivity of PDB $\left(C_{P}=2 r_{12} /\right.$ $\left.r_{14}^{*}=2 r_{13} / r_{15}^{*}=0.32\right)$ as reported in other works on this field. ${ }^{[5]}$ This modified set of kinetic parameters was used in the remaining predictions here presented.

In this work, the priority was the description of semi-batch operation, with a main goal being the prediction of the polymerization behavior using the same set of kinetic parameters as for batch operation and this was achieved, as shown in Figure 3(a). Figure 3(b) shows the comparison of measured and predicted values of $\bar{M}_{n}, \bar{M}_{w}$ and $\bar{M}_{z}$ for a semi-batch run. A good agreement is obtained for $\bar{M}_{w}$ (which

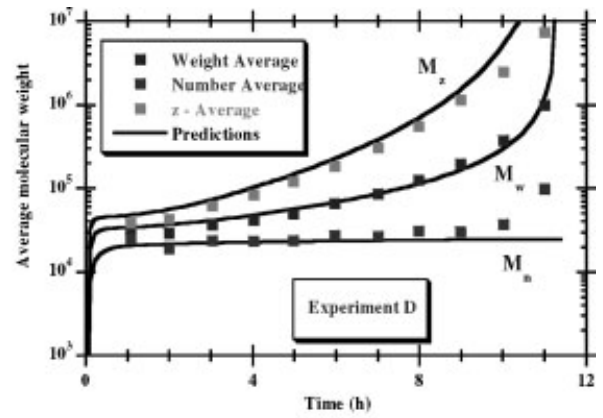

(b)

Figure 3.

Predicted and measured $\bar{M}_{w}$ for linear and non-linear polymerization systems in batch and semi-batch reactor (a). Time evolution of $\bar{M}_{n}, \bar{M}_{w}$ and $\bar{M}_{z}$ during the radical copolymerization of S/DVB in semi-batch reactor using different feed policies. 
is the molecular mass directly measured by MALLS) but significant deviations are observed for $\bar{M}_{n}$ and $\bar{M}_{z}$ close to gel point when a high dispersion of molecular sizes occurs. This is a consequence of the approximations involved in the indirect estimation of $\bar{M}_{n}$ and $\bar{M}_{z}$ which considers a homogeneous polymer population inside each SEC slice. Inaccurate measurements for $\bar{M}_{n}$ and $\bar{M}_{z}$ are thus obtained using SEC/MALLS for highly polydispersed polymers.

Figure 4(a) shows the comparison of batch operation including a CTA and different feed policies of DVB to a semi-batch reactor. These results show that operation in semi-batch reactor can be used as an alternative to the inclusion of a CTA in batch operation, namely for producing soluble branched polymers at higher monomer conversions.

The time evolution of the experimental $z$-average mean-square molecular radius of gyration $\left(\bar{R}_{g}\right)$ in THF solution as obtained by MALLS is presented in Figure 4(b). For the smaller molecular dimensions approaching $10 \mathrm{~nm}$, higher experimental errors are observed as the lower limit of the instrument detection is attained.

Our theoretical predictions of $\bar{R}_{g}$ are only valid for Gaussian chains, described as a set of beads connected by freely rotating rods of length $b$, estimated as $b=0.692 \mathrm{~nm}$ from the experimental $\bar{R}_{g, l i n_{\theta}}=0.0277 M^{0.5} \mathrm{~nm}$ with a $\Theta$ solvent (trans-decaline at $\left.22^{\circ} \mathrm{C}\right) .{ }^{[22]}$

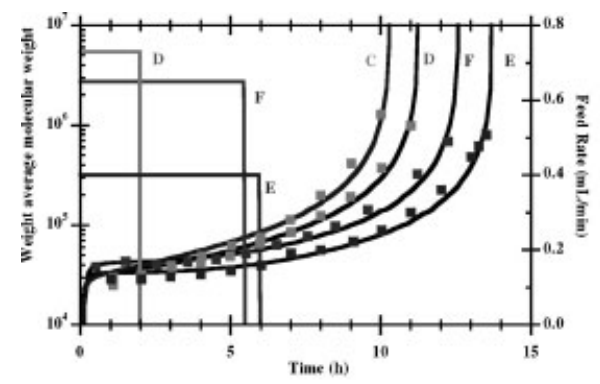

(a)
For polystyrene in THF, $R_{g, \text { lin }}=$ $0.0118 M^{0.6} \mathrm{~nm}$ at $25^{\circ} \mathrm{C} .{ }^{[22]}$ Neglecting the small temperature differences, we estimate an expansion factor of the gyration radius for linear polydispersed polystyrene in THF at $30^{\circ} \mathrm{C}$ as $\bar{R}_{g, l i n} / \bar{R}_{g, l i n_{\theta}}=0.426 \bar{M}_{z}^{0.1}$.

Since an extension of our method for taking into account the presence of the excluded volume effect has not yet been developed ${ }^{[10]}$ we have assumed equality of the expansion factors for branched and linear polymer molecules for obtaining the predictions in Figure 4(b). This assumption has been previously used by several researchers ${ }^{[23]}$ and has not been questioned in more recent works with similar goals as ours. Note that Monte Carlo simulation and molecular dynamics have gone a long way to describe real chains and even branched ones, ${ }^{[2]}$ but the complexity of kinetic schemes such as the one here discussed precludes the direct use of approaches which have successfully tackled simpler structures such as comb and star polymers.

A polymer population with large dimensions but at a low concentration is detected by SEC-MALLS close to gel point, as presented in Figure 5. Figure 6(a) shows the chromatogram of an S/DVB sample collected close to gel point. A molecular fraction with a strong light scattering signal but a weak refractive index response is easily identified. Figure 6(b) compares chromatograms of samples synthesized at different conditions. In the presence of

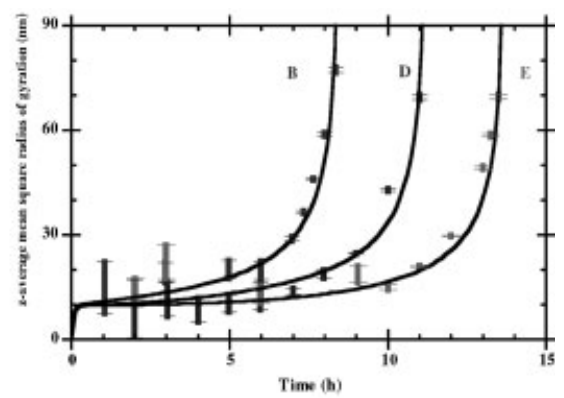

(b)

Figure 4 .

Time evolution of $\bar{M}_{w}$ in the presence of CTA or using different feed policies in semi-batch reactor (a). Time evolution of $\bar{R}_{g}$ in THF solution during the radical copolymerization S/DVB in batch or semi-batch reactor (b). 


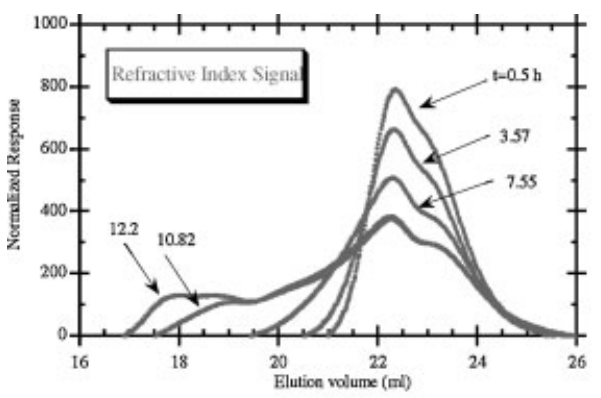

(a)

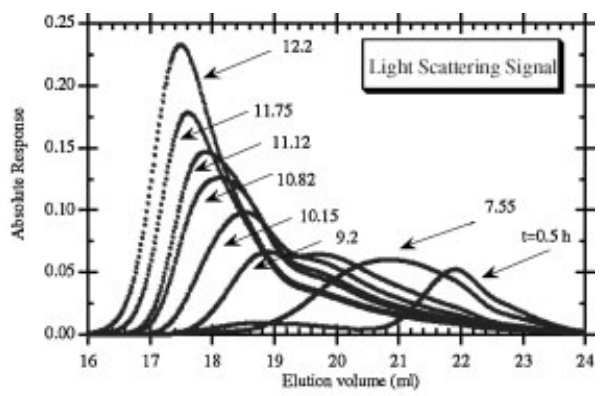

(b)

Figure 5 .

Measured refractive index signal (a) and $90^{\circ}$ light scattering signal (b) in the SEC chromatograms of samples of S/DVB corresponding to different polymerization times in a semi-batch reactor.

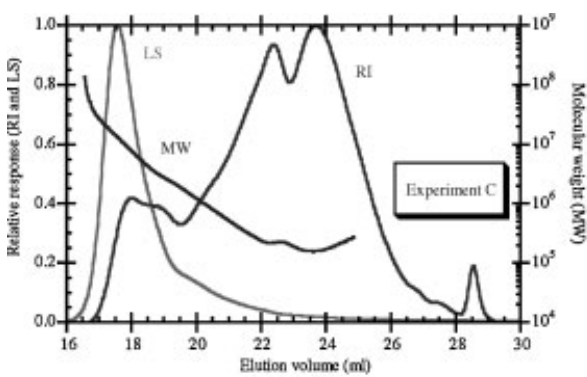

(a)

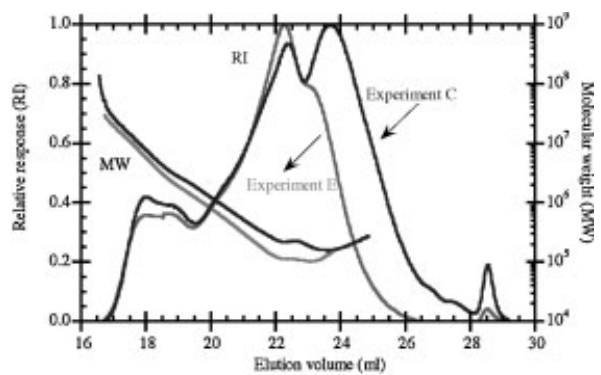

(b)

Figure 6.

Molecular weight along the SEC chromatogram for samples of S/DVB synthesized using different operation conditions. In both cases the polymerization time is very near to gel point.

CTA (experiment $\mathbf{C}$ ) a high polydispersity of molecular sizes is observed. In both cases, an inversion in the relation MW vs. elution volume is observed, confirming the

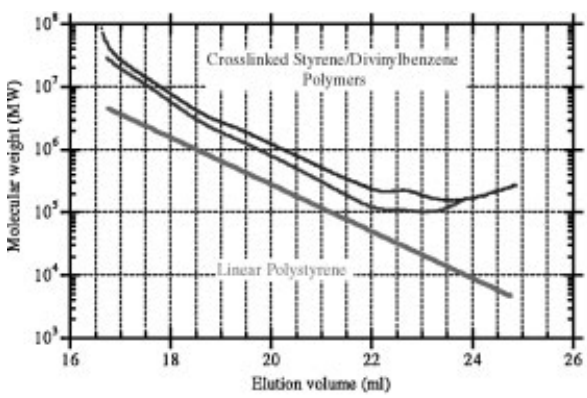

(a) erroneous interpretation of chromatograms of branched polymers which would be caused from using a calibration with linear polymers, as seen in Figure 7(a). Similar

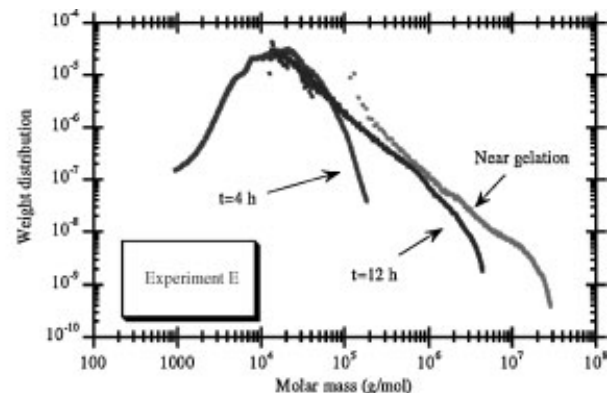

(b)

Figure 7.

Observed relations molecular weight vs. elution volume for S/DVB (a). Time evolution of the molecular weight distribution of S/DVB synthesized in semi-batch reactor showing the formation of a long end tail as gelation is approached (b). 
observations have been reported in the literature. ${ }^{[4]}$

Note that in the interpretation of chromatograms such as the one presented in Figure 6(a) the lower limit of light scattering detection must be accounted for and a small region at the right side cannot be quantitatively analyzed. Figure 7(b) shows the time evolution of the molecular weight distribution of branched S/DVB: the formation of a long end tail as gelation is approached agrees with previous theoretical results. ${ }^{[25]}$

\section{Conclusions}

Using styrene + divinylbenzene as a model system, the radical copolymerization of mono- and divinyl- monomers has been experimentally studied in a semi-batch reactor. It has been shown that the production of branched/crosslinked polymers with an improved control of molecular architecture can be carried out by adjusting the feed policy of the divinyl monomer. These results can be especially useful to obtain soluble branched polymers at higher conversions than with a conventional batch operation. A detailed kinetic model taking into account some complexities of these polymerization systems has been developed in the absence of intramolecular reactions. These modeling studies can be used to design new feed policies with impact on the properties of the synthesized materials. It has also been shown that the interpretation of the SEC chromatograms of branched polymers is complicated due to the possible change in the relation between molecular weight and elution volume (or hydrodynamic radius), owing to the existence of copolymer chains with the same molecular weight but quite different molecular sizes (elution volumes).

The guidelines here presented pave the way to further kinetic studies of this or similar chemical systems with inclusion of cyclization reactions and others (such as. methyl methacrylate + ethylene glycol dimethacrylate) with suspected effects of spatial segregation of chemical groups. The extension of the present study to living or mediated non-linear systems ${ }^{[26]}$ is another application with potential interest.

Acknowledgements: Financial support by Fundação para a Ciência e a Tecnologia (FCT), Ministry of Science and Technology of Portugal and European Community through FEDER (projects POCI/EQU/ 44784/2002 and POCI/EQU/60483/2004) is gratefully acknowledged. We thank undergraduate students Ana Dias, Isabel Sousa, Sandra Curralo and Sónia Soares for their help in the experimental work.

[1] S. P. Gretton-Watson, E. Alpay, J. H. G. Steinke, J. S. Higgins, Chem. Eng. Sci. 2006, 61, 1421.

[2] G. Saunders, P. A. G. Cormack, S. Graham, D. C. Sherrington, Macromolecules 2005, 38, 6418.

[3] N. O'Brien, A. McKee, D. C. Sherrington, A. T. Slark, A. Titterton, Polymer. 2000, 41, 6027.

[4] I. Bannister, N. C. Billingham, S. P. Armes, S. P. Rannard, P. Findlay, Macromolecules. 2006, 39, 7483.

[5] O. Okay, Prog. Polym. Sci. 2000, 25, 711.

[6] G. Bayramoglu, F. B. Senkal, G. Celik, M. Y. Arica, Colloids and Surfaces A 2007, 294, 56.

[7] R. C. S. Dias, M. R. P. F. N. Costa, Polymer 2005, 46, 6163.

[8] M. R. P. F. N. Costa, R. C. S. Dias, Chem. Eng. Sci. 2005, 60, 423.

[9] R. C. S. Dias, M. R. P. F. N. Costa, Polymer 2006, 47, 6895 .

[10] M. R. P. F. N. Costa, R. C. S. Dias, Polymer 2007, 48, 1785.

[11] G. Moad, D. H. Solomon, "The Chemistry of Radical Polymerization”, Elsevier, 2006.

[12] A. K. Nyhus, S. Hagen, A. Berge, J. Polym. Sci. 1999, 37, 3345 .

[13] M. Hecker, "Experimentelle Untersuchungen und Monte-Carlo-Simulation netzwerkbildender Copolymerisationen." Fortschritte der Polymerisationstechnik II (H.U. Moritz ed.) Wissenschaft \& Technik Verlag Berlin, ISBN 3-89685-353-8, 2000.

[14] O. Okay, D. Kaya, O. Pekcan, Polymer. 1999, 40, 6179.

[15] J. C. Bevington, Trans. Faraday Soc. 1955, 51, 1392. [16] M. Buback, R. G. Gilbert, R. A. Hutchinson, B. Klumperman, F. D. Kuchta, B. G. Manders, K. F. O'Driscoll, G. T. Russel, J. Schweer, Makromol. Chem. Phys. 1995, 196, 3267.

[17] J. Brandrup, E. H. Immergut, E. A. Grulke, A. Abe, D. R. Bloch, "Polymer Handbook" (4 ${ }^{\text {th }}$ Edition). John Wiley \& Sons, 1999.

[18] G. Odian, "Principles of polymerization" (4th Edition). Wiley-Interscience, New York 2004. 
[19] M. S. Matheson, E. E. Auer, E. B. Bevilacqua, E. J. Hart, Macromolecules. 1951, 73, 1700.

[20] S. Beuermann, M. Buback, Prog. Polym. Sci. 2002, 27, 191.

[21] M. R. P. F. N. Costa, R. C. S. Dias, Macromol. Theory Simul. 2003, 12, 560.

[22] K. Terao, J. W. Mays, Eur. Polym. J. 2004, 40, 1623.
[23] Z. Dobkowski, J. Appl. Polym. Sci. 1985, 30, 355. [24] M. O. Steinhauser, J. Chem. Phys. 2005, 122, 094901.

[25] R. C. S. Dias, M. R. P. F. N. Costa, Macromol. Theory Simul. 2005, 14, 243.

[26] Y. Saka, P. B. Zetterlund, M. Okubo, Polymer. 2007, 48, 1229. 